\title{
Modelling chemical vapour infiltration of pyrolytic carbon as moving boundary problem
}

\author{
T.-A. Langhoff*, E. Schnack \\ Institute of Solid Mechanics, Karlsruhe University, Kaiserstrasse 12, 76128 Karlsruhe, Germany
}

\section{A R T I C L E I N F O}

\section{Article history:}

Received 4 February 2006

Received in revised form 1 April 2008

Accepted 11 April 2008

Available online 4 May 2008

\section{Keywords:}

Chemical vapour infiltration

Mathematical modelling

Moving boundary problem

Materials processing

\begin{abstract}
A B S T R A C T
Chemical vapour infiltration (CVI) of pyrolytic carbon is described as a moving boundary problem to determine the evolution of the pyrolytic carbon layer in space and time. Derived from real geometries, a one-dimensional single pore model is developed yielding a nonlinear coupled system of partial differential equations for the concentrations of the gas phase species and the height of the carbon layer within cylindrical pores. The evolution of the moving boundary of the gas phase domain is governed by a non-differentiable minimisation condition. Additionally, a CVI reactor model to describe the infiltration of several cylindrical pores within a porous substrate is presented on the basis of the single pore model. Both models are new in that they combine the following features: (i) derivation of the equations rigorously taking into account the temporal change of the gas phase, (ii) the explicit construction of the position of the gas-solid interface, (iii) the influence of the local curvature of the carbon layer on its growth velocity, and (iv) modelling of chemical kinetics using a reduced reaction scheme with intermediate gas phase species and several surface reactions. The models are solved numerically using a staggered strongly decoupled scheme with implicit Euler time integration. The results allow the identification of process conditions and geometries for which a complete infiltration of the pores or the whole substrate is achieved. For low pressures, the predictions of the CVI reactor model are in agreement with the available experimental data.

(c) 2008 Elsevier Ltd. All rights reserved.
\end{abstract}

\section{Introduction}

One important industrial process for producing carbon fibre reinforced carbon (CFC) is chemical vapour infiltration (CVI). Formally, gaseous carbon is deposited on the surface of a porous substrate due to the complex interaction of homogeneous gas phase and heterogeneous surface reactions (Hüttinger, 1998). Additionally, convection and diffusion also have a significant influence on the deposition of pyrolytic carbon on the substrate (Middleman, 1989). CVI is a very slow and thus expensive technique; consequently, there is a huge need in process optimisation based on detailed mathematical modelling and precise and efficient numerical simulations. Many different variants of CVI exist: isothermal (Reuge and Vignoles, 2005; Sotirchos, 1991; Zhang and Hüttinger, 2001; Jin and Wang, 2000, 2002, 2003; McAllister and Wolf, 1991; Chung et al., 1991), thermal gradient CVI (Tago et al., 2001) forced flow CVI (Probst et al., 1999; Roman et al., 1995; microwave heating, Morell et al., 1993;

\footnotetext{
* Corresponding author. Tel.: +497216086905; fax: +497216084187.

E-mail address: langhoff@itm.uni-karlsruhe.de (T.-A. Langhoff).
}

Midha and Economou, 1998), etc. In this work we concentrate on the isothermal isobaric CVI of pyrolytic carbon since experimental data are available for this process.

The main aim in modelling CVI is the determination of the evolution of the gas-solid interface in time and space. The mathematical formalisation of this complex physical and chemical process results in a moving boundary problem and accounts for detailed chemical reaction kinetics that in combination with different transport mechanisms in the gas phase affect the deposition of pyrolytic carbon. Although CVI studies have dealt with quite complex preform geometries (see e.g. Sotirchos, 1991; Tago et al., 2001), the principal understanding of the interaction of the different processes is still not completely clear. Therefore principal studies using simplified geometries are necessary in order to finally achieve an optimisation of the whole CVI process even for complex geometries.

Many of the published works dealing with CVI are based on a simplified reaction kinetics and consist of only one overall reaction (Sotirchos, 1991; McAllister and Wolf, 1991; Chung et al., 1991; Tago et al., 2001; Roman et al., 1995; Morell et al., 1993; Midha and Economou, 1998; Hou et al., 1999). The processes in the gas phase are often assumed steady-state (Middleman, 1989; Reuge and Vignoles, 2005; Zhang and Hüttinger, 2001; McAllister and Wolf, 
1991; Chung et al., 1991; Tago et al., 2001; Hou et al., 1999) which is critical since the temporal evolution of the geometry due to deposition influences the transport mechanisms within this phase. At a first glance, the values of the so-called condensation ratio (the ratio of the density and the mass of the solid product and the concentration of the gas-phase species) being superior to $10^{4}$ might seem to support the assumption of a steady-gas phase by many authors. However, the pure value of the condensation ratio cannot capture all effects from the movement of the boundary on the transport and reaction processes. The properties of the composite material obtained by CVI are directly related to the spatial distribution of the deposited material. The question of complete infiltration of the given porous substrate is thus of significant importance. In many studies, porosities are calculated (Reuge and Vignoles, 2005; McAllister and Wolf, 1991; Tago et al., 2001; Morell et al., 1993; Midha and Economou, 1998; Hou et al., 1999; Kulik et al., 2004), but the position of the interface is not tracked. Consequently, the question whether the given substrate is completely infiltrated or not remains open.

In a series of papers (Jin and Wang, 2000, 2002, 2003; Jin et al., 1999) the level set equation is coupled to a Laplace equation for the concentrations to track the gas-solid interface. However, the gas phase is assumed as steady-state, constant diffusion coefficients are used and no gas phase reactions are considered. A precise interface front tracking in a complex porous medium has also been described in Vignoles (2006), similar to Jin and Wang $(2000,2002,2003)$ but using a different numerical method. The growth rate of the deposited carbon layer does not only depend on the total deposition rate of solid pyrolytic carbon, but also on the local curvature of the carbon layer. To the knowledge of the authors, the CVI models in Jin and Wang (2002) and Merz and Rybka (2004) have been so far the only ones taking into account the local curvature of the carbon layer in calculating the growth velocity.

Cylindrical pores are used as idealised geometries in different CVI-studies (Middleman, 1989; Zhang and Hüttinger, 2001; Tago et al., 2001; Roman et al., 1995; Hou et al., 1999). More complicated geometries have also been considered: a network of uniformly sized cylindrical capillaries (Sotirchos, 1991), a system of plies consisting of woven tows of bundles of filaments (Chung et al., 1991), and a random packing of fully overlapping capillaries (Reuge and Vignoles, 2005; Morell et al., 1993), and a random pore model (McAllister and Wolf, 1991). The evolution of such complex geometries is mostly governed in dependence of the porosity of the preform. In Kulik et al. (2004), two systems of parallel pores were chosen: pores within a fibre bundle and between different parallel fibre bundles. It was shown that the convective mass transport between these two systems greatly influences the porosity evolution and the infiltration times. Additionally, species concentration profiles along the preform thickness were computed. However, whenever treating a complex geometry, no attempt has been made to determine the precise location of the interface. Consequently, these models do not answer the question of complete infiltration of a given substrate.

Approaches have been performed in direction of optimising the CVI process (Chang et al., 1998; Ditkowski et al., 2000), but only one overall chemical reaction is treated and the underlying differential equations are based on steady-state assumptions both for the concentrations as well as for the transport coefficients. However, no mathematical optimisation techniques are used. For isobaric and isothermal CVI and one spatial dimension asymptotic analysis is used in Jones (2006) to derive criteria for process parameters in order to assure that the remaining voids are within a given tolerance. In Merz and Rybka (2004) the existence and uniqueness of a local solution of a free boundary problem was proved. However, constant diffusion coefficients, reaction rates being linear in the concentrations and the Gibbs-Thompson law for the movement of the free boundary were assumed. The problem of the spatiotemporal surface evolution of any particular surface for the given normal velocity field has also been treated using generalised kinematic models (Katardjiev et al., 1994). This approach results in the nonlinear hyperbolic Hamilton-Jacobion equations representing Huygens principle of wavefront propagation. Due to the a priori given velocity this approach cannot be used when the surface evolution interacts with flow or transport processes as e.g. in CVI. A similar problem occurs in modelling the shape evolution in trenches (Georgiadou and Veyret, 2002) or in electrochemical machining (Domanowski and Kozak, 2001). In Ross (1988) the mathematical theory of hyperbolic conservation laws has been applied to the process of ion etching in order to determine the evolution of material boundaries.

Recently, reduced reaction schemes for describing the gas phase chemistry in chemical vapour deposition (CVD) of pyrolytic carbon from methane were published and compared with experimental results (Hüttinger, 1998; Benzinger et al., 1996; Becker and Hüttinger, 1998). They include composition reactions in the gas phase yielding intermediate gas phase species as well as several reactions for carbon formation from different gas phase species. The influence of back-mixing on the decomposition of methane has been studied in Andrä et al. (2001).

The new aspect of the present paper is the modelling of CVI of pyrolytic carbon combining all the following fundamental features:

- set up as moving boundary problem rigorously taking into account the temporal change of the gas phase and of the solid phase as well as all their consequences;

- explicit determination of the temporal and spatial evolution of the gas-solid interface;

- consideration of the local curvature as factor in the growth velocity of the carbon layer in addition to the total deposition rate of pyrolytic carbon;

- numerical evaluation of the influence of the curvature term on the simulated deposition profiles;

- description of the gas phase chemistry using not only one single overall reaction, but also a reduced reaction scheme with several intermediate gas phase species and different deposition reactions to form solid pyrolytic carbon.

A single pore model to describe the infiltration of a single cylindrical pore is developed consisting of a nonlinear convection-diffusionreaction system for the concentrations of the gas phase species coupled to a nonlinear evolution equation for the height of the carbon layer. Based on this single pore model, a CVI reactor model is set up. The CVI reactor-model consists of a convection-reaction system coupled to the systems of several single pore models via Dirichlet boundary conditions. The choice of such a simplified geometry can be justified by two reasons: first, the aim of this study is to describe the fundamental processes in CVI correctly and not to develop a huge and complicated model (due to complex geometries). Secondly, experimental data were available precisely for such geometries (Hu and Hüttinger, 2001) allowing the necessary verification of the CVI reactor model. The models presented in this manuscript are sharp-interface models for tracking the moving boundary on the fibre-scale. In this form they do not apply to the macroscopic scale of the complete composite for which homogenisation procedures have to be applied. The paper is organised as follows. In Section 2, both the single pore model and the CVI reactor model are derived and presented in detail. The numerical solution procedure is described as well as the reaction scheme used. In Section 3, the results are presented as well as compared to experimental data. 


\section{Mathematical mode}

\subsection{A single pore model}

Studying CVI of fibre bundles or even fibre felts we are dealing with very complex geometries challenging both the theoretical framework and the numerical simulations to be carried out.

In Fig. 1(a) a part of an infiltrated fibre felt is shown. The original shape of the single carbon fibres (with typical values of the diameter around $10 \mu \mathrm{m}$ ) can be distinguished from the deposited solid pyrolytic carbon. Fig. 1(b) shows a view on an infiltrated bundle of unidirectional carbon fibres. In Figs. 1(a) and (b), the dark parts are regions that remained uninfiltrated during the CVI process.

To gain more knowledge about the important processes (mass transport and reactions) and their complex interaction within a changing geometry in CVI of pyrolytic carbon, the geometries to be considered have to be idealised. In a first approximation, we assume that the pores that remained uninfiltrated have a cylindrical shape.

In the following, a single pore model to study the infiltration of a single cylindrical pore with initial diameter $d_{p}^{0}$ and initial length $l_{0}$ (see Fig. 2) is developed. In a first step, according to the assumptions about the geometry, the domains are identified. Secondly, the evolution of the moving boundary is derived. In a third step, the equations to describe the processes in the gas phase are derived. Finally, to complete the model, initial and boundary conditions are given.

As can be seen from Fig. 2, the open pore mouth constitutes a fixed boundary $\Gamma_{\text {fixed }}$, whereas the evolution of the moving boundary $\Gamma(t)$ between the gas phase $\Omega(t)$ and the solid phase $\tilde{\Omega}(t)$ is wanted. Assuming cylindrical symmetry and a small aspect ratio, i.e. $d_{p}^{0} \ll l_{0}$, a spatially one-dimensional model can be set up with the spatial coordinate $x$ describing the distance from the pore entrance (see Fig. 3). At each position $x<l_{0}$ and at each time point $t>0, h(t, x)$ denotes the height of the carbon layer that has already been deposited on the pore wall and $d_{p}(t, x)$ denotes the remaining free diameter of the pore. The opening of the pore moves to positions with $x<0$; however, this is neglected in the single pore model since the conditions for deposition might be completely different outside the pore (ratio of volume to surface area, transport processes, etc.) and will additionally depend on details of the geometry considered outside of the pore.

Then, the domain of the gas phase is given by an interval

$\Omega(t)=\{x \in \mathbb{R}: 0 \leqslant x \leqslant l(t)\}, \quad t \in\left[0 ; t_{\max }\right]$ with constant left border at $x=0$ and varying right border $l(t)$ which denotes the actual depth of the pore. $t_{\max }$ is the maximal duration of the infiltration process.

In this problem, in principle, the moving boundary has to be tracked along two directions: (i) along the radial direction, it is the height of the carbon layer yielding the position of the moving boundary, and (ii) in $x$-direction constraining the depth of the pore. However, due to the small aspect ratio, we can neglect any deposition on the pore ground and restrict ourselves to deposition on the pore walls. For clarity, the justification of this neglection can be found later in more details when the complete model (including the

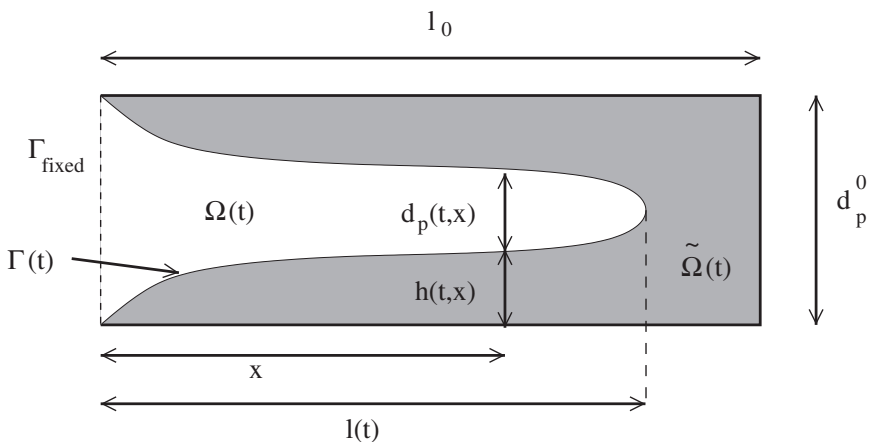

Fig. 2. Schematic view of a single cylindrical pore of initial radius $r_{0}$ and initial length $l_{0}$.

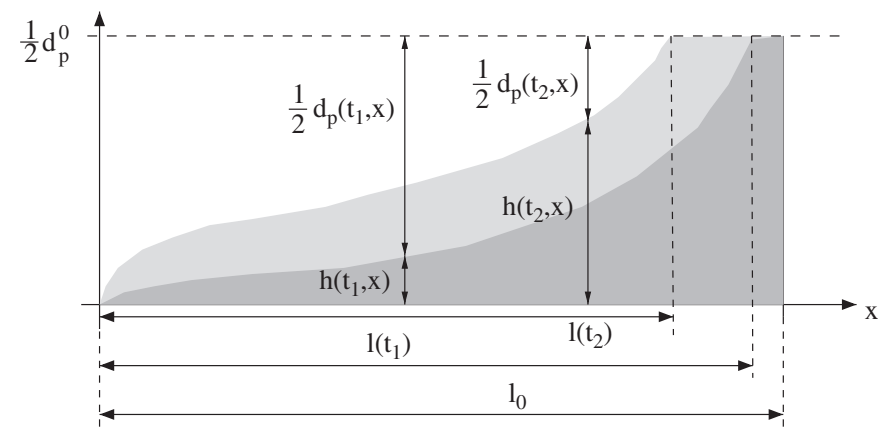

Fig. 3. Domains and height of the carbon layer in the one-dimensional model for two time instances $0<t_{1}<t_{2}$.
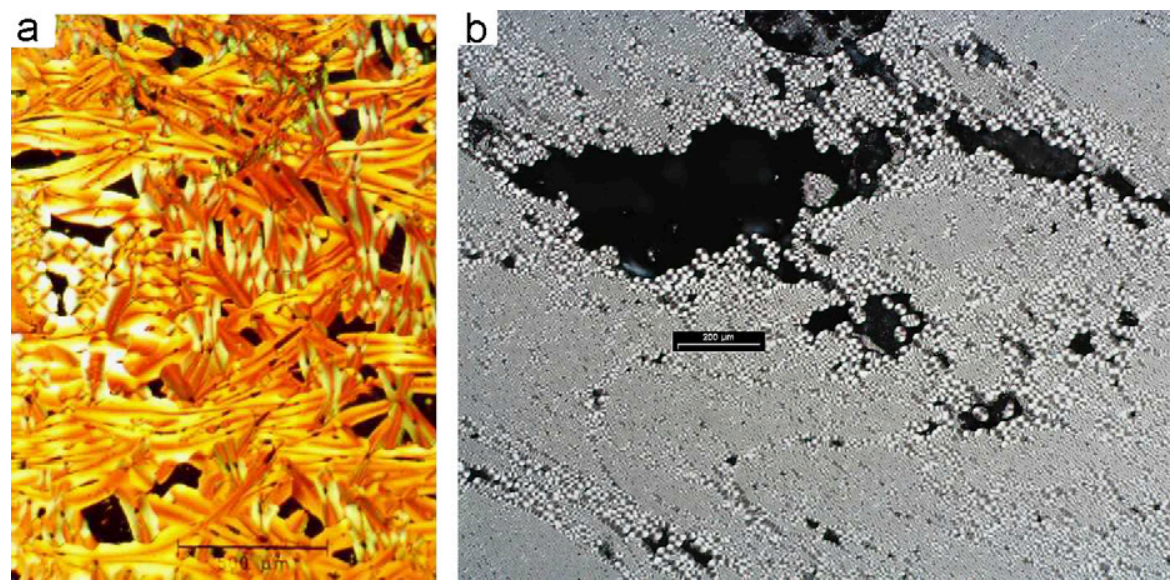

Fig. 1. (a) Infiltrated fibre felt (given scale: $500 \mu \mathrm{m}$ ) and (b) infiltrated bundle of unidirectional carbon fibres (given scale: $200 \mu \mathrm{m}$ ). 
description of the chemistry) has been set up. Thus, for each time $t$ the location of the moving boundary $\Gamma(t)$ is only determined by $h(t, x)$. The process of pore closing appears if, at some time $t$ and some point $x \in \Omega(t)$, the height of the carbon layer $h(t, x)$ reaches the original pore radius. Thus the time dependence of $l(t)$ is given by

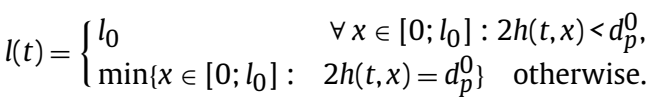

Thus, $l(t)$ measures the distance from the pore entrance to the first of all possible points where the pore is closed. As can be directly concluded from the non-differentiable minimisation condition (2), $l(t)$ needs not even be continuous. Note that the domain of the gas phase $\Omega(t)$ is a function of $l(t)$. The evolution of the gas phase can only be determined by computing the distribution of the height of the carbon layer, $h(t, x)$, within the whole gas phase domain.

Having defined the domain of the gas phase, $\Omega(t)$, the processes that occur within the gas phase can now be described mathematically. Convection is neglected within the pore due to the small aspect ratio. Thus diffusion is the only transport mechanism to be considered together with the chemical reactions of the chemical species. Since methane is the dominant species in the gas phase we only take into account binary diffusion between each gas phase species and methane. Additionally, we consider Knudsen diffusion and combine both types into an effective diffusion coefficient in the following way:

$D_{i}(t, x)^{-1}=D_{i}^{\text {bin }^{-1}}+D_{i}^{K}(t, x)^{-1}, \quad i=1, \ldots, N_{g s}$,

with $N_{g s}$ denoting the number of gas phase species. The binary diffusion coefficients are calculated using the Chapman-Enskog equation:

$D_{i}^{\text {bin }}=1.8583 \times 10^{-3} \frac{T^{3 / 2}}{p \sigma_{i}^{2} \Omega} \sqrt{\frac{M_{i}+M_{\mathrm{CH}_{4}}}{M_{i} M_{\mathrm{CH}_{4}}}}$.

Here, $M_{i}$ denotes the molecular mass of species $i, T$ denotes the absolute temperature, $p$ denotes the pressure, $\sigma_{i}^{2}$ is the mean collision diameter, and $\Omega$ is the collision integral. In this model of isothermal CVI, the binary diffusion coefficients are constant for each gas phase species. The Knudsen diffusion coefficients depend on the free pore diameter $d_{p}$ and are given by

$D_{i}^{K}(t, x)=\frac{d_{p}(t, x)}{3} \sqrt{\frac{8 R T}{\pi M_{i}}}, \quad(t, x) \in\left[0 ; t_{\max }\right] \times \Omega(t)$.

Since the gas phase domain is explicitly time dependent, the diffusion-reaction system for the concentrations within the gas phase cannot be obtained in the standard form. Based on the assumption of Fick's second law, the diffusive fluxes, $j_{i}^{d}$, for the different gas phase species are given by

$j_{i}^{d}=-D_{i}(t, x) \frac{\partial c_{i}}{\partial x}(t, x), \quad i=1, \ldots, N_{g s}$.

The generalised mole balance equation for a volume element like the one shown in Fig. 4 consists of contributions due to the change of volume, due to reactions between the species considered and due to the diffusive fluxes through the surfaces at $x$ and at $x+\Delta x$ during the time increment $\Delta t$ :

$$
\begin{aligned}
& c_{i}(t+\Delta t, x) \Delta V(t+\Delta t, x)-c_{i}(t, x) \Delta V(t, x) \\
& \quad=r_{i}(t, x, \mathbf{c}) \Delta V(t, x) \Delta t+j_{i}^{d}(t, x) a(t, x) \Delta t-j_{i}^{d}(t, x+\Delta x) a(t, x+\Delta x) \Delta t,
\end{aligned}
$$

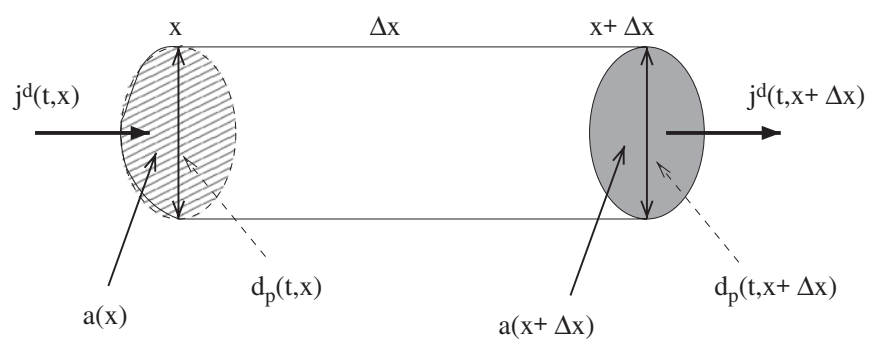

Fig. 4. Geometry of a small volume element between $x$ and $x+\Delta x$ with varying circular cross section.

where the area of the cross section is given by

$a(t, x)=\frac{\pi}{4} d_{p}^{2}(t, x)$

Division of Eq. (7) by $\Delta x \cdot \Delta t$, using Eq. (8) and $\Delta V(t, x)=\Delta x \cdot a(t, x)$ we obtain in the limit $\Delta x \rightarrow 0, \Delta t \rightarrow 0$

$\frac{\partial}{\partial t}\left[a(t, x) c_{i}(t, x)\right]=\frac{\partial}{\partial x}\left[a(t, x) D_{i}(t, x) \frac{\partial c_{i}}{\partial x}(t, x)\right]+a(t, x) r_{i}(t, x, \mathbf{c})$

with $r_{i}$ denoting the reaction rates of gas phase and deposition reactions of gas phase species $i=1, \ldots, N_{g s}$. Modelling the processes within the gas phase region behind a closing point of the pore is not intended here and would have to involve different equations.

Now the evolution of the height of the carbon layer has to be derived. The growth velocity $v_{\Gamma}$ of this pyrolytic carbon layer normal to the surface is obtained as

$v_{\Gamma}(t, x)=\frac{M_{\mathrm{C}_{\infty}}}{\varrho_{\mathrm{C}_{\infty}}} r_{\mathrm{C}_{\infty}}(t, x), \quad(t, x) \in\left[0 ; t_{\max }\right) \times \Omega(t)$

where $r_{C_{\infty}}$ denotes the total production rate for the formation of solid pyrolytic carbon due to the heterogeneous deposition reactions and $M_{\mathrm{C}_{\infty}}$ and $\varrho_{\mathrm{C}_{\infty}}$ are the molecular mass and density of solid pyrolytic carbon, respectively. Taking into account the local curvature of the deposited carbon layer (see Fig. 9), it is only the component of $v_{\Gamma}(t, x)$ in direction of $h(t, x)$ that affects the growth of the carbon layer (since we neglected deposition on the pore ground). As well known (Sethian, 1985), the angle $\alpha$ is given by the slope of the carbon layer resulting in a nonlinear equation for the height of the carbon layer: for $(t, x) \in\left(0 ; t_{\max }\right) \times \Omega(t)$

$\frac{\partial}{\partial t} h(t, x)=\frac{M_{\mathrm{C}_{\infty}}}{\varrho_{\mathrm{C}_{\infty}}}\left(1+\left(\frac{\partial h}{\partial x}(t, x)\right)^{2}\right)^{-1 / 2} r_{\mathrm{C}_{\infty}}(t, x, \mathbf{c})$.

The consideration of curvature for the evolution of surfaces or fronts occurs in many fields and can be found e.g. in Sethian (1985) and references therein.

If the deposition on the pore ground was not neglected, another equation similar to Eq. (11) and instead of Eq. (2) would have been necessary to describe the evolution of the boundary.

Using the purely geometrical consideration (see Figs. 2 and 3)

$2 h(t, x)+d_{p}(t, x)=d_{p}^{0}$,

the system of Eq. (9) can be transformed finally containing only the concentrations of the gas phase species and the height of the carbon layer as unknown. To achieve this, we substitute Eqs. (3), (5), 
(8), (11) and (12) in Eq. (9) and obtain $\left(i=1, \ldots, N_{g s}\right)$ :

$$
\begin{aligned}
\frac{\partial c_{i}}{\partial t}(t, x)= & \frac{4 M_{C_{\infty}}}{\varrho C_{\infty}} \frac{r_{C_{\infty}}(t, x, \mathbf{c})}{d_{p}^{0}-2 h(t, x)}\left(1+\left(\frac{\partial h}{\partial x}(t, x)\right)^{2}\right)^{-1 / 2} c_{i}(t, x) \\
& +6 D_{i}^{\text {bin }} \frac{3 D_{i}^{\text {bin }} \sqrt{\frac{\pi M_{i}}{8 R T}}+2\left(d_{p}^{0}-2 h(t, x)\right)}{\left(3 D_{i}^{\text {bin }} \sqrt{\frac{\pi M_{i}}{8 R T}}+d_{p}^{0}-2 h(t, x)\right)^{2}} \frac{\partial h}{\partial x}(t, x) \frac{\partial c_{i}}{\partial x}(t, x) \\
& +3 D_{i}^{\text {bin }} \frac{d_{p}^{0}-2 h(t, x)}{3 D_{i}^{\text {bin }} \sqrt{\frac{\pi M_{i}}{8 R T}}+d_{p}^{0}-2 h(t, x)} \frac{\partial^{2} c_{i}}{\partial x^{2}}(t, x) \\
& +r_{i}(t, x, \mathbf{c}), \quad \forall(t, x) \in\left(0 ; t_{\max }\right) \times \Omega(t),
\end{aligned}
$$

showing the structure of a nonlinear convection-diffusion-reaction system. The diffusion coefficients, the velocity, and the reactive contributions are all varying in time and space. Together with the evolution equation for the carbon layer, Eq. (11), this system is to be solved for the concentrations and the height of the carbon layer. To the knowledge of the authors, systems (11) and (13) to describe the processes in the gas phase and the growth of the deposited layer is used for the first time in studies about modelling CVI.

To complete the model, the following boundary conditions are chosen:

$c_{i}(t, x=0)=c_{i}^{0}$

$\frac{\partial c_{i}}{\partial x}(t, x=l(t))=0 \quad t \geqslant 0, i=1, \ldots, N_{g s}$

with given concentrations at the pore entrance, $c_{i}^{0}$. As initial conditions, the concentrations for all species within the pore and at the pore depth are set to zero as well as the height of the carbon layer within the whole pore.

$c_{i}(t=0, x)=0, \quad 0<x \leqslant l_{0}, \quad i=1, \ldots, N_{g s}$,

$h(t=0, x)=0, \quad 0 \leqslant x \leqslant l_{0}$.

\subsection{A CVI reactor model}

For describing the infiltration of porous substrates containing several pores (see Fig. 10) a CVI reactor model is developed on the basis of the single pore model described above. Using this model the influence of the location of the pores within the substrate as well as that of the convection of the gas mixture on the deposition profiles within the pores can be studied.

Within the plane substrate, $N_{p}$ pores are assumed to be of cylindrical shape and perpendicular to the substrate surface. Domain decomposition of the total gas phase domain (see Fig. 10) distinguishes the domain outside of the pores, $\Omega_{\text {out }}$, from the domains within the different pores $\Omega_{j}(t), j=1, \ldots, N_{p}$ :

$\Omega(t)=\Omega_{\text {out }} \cup \bigcup_{j=1}^{N_{p}} \Omega_{j}(t)$.

Outside of the pores, deposition of pyrolytic carbon at the reactor walls can be neglected due to the dimensions of the reactor. This is the reason why $\Omega_{\text {out }}$ does not depend on time. In $\Omega_{\text {out }}$, diffusion is neglected because convection is dominating. The Peclet numbers for $\Omega_{\text {out }}$ range from 3.2 up to 4.6, depending on the process parameters $T$ and $p$. As the pore diameter is very small compared to the length scale outside of the pores, the flow into or out of the pores as well as the mass loss in $\Omega_{\text {out }}$ can be neglected. The convection velocity in $\Omega_{\text {out }}$ is assumed to be constant. Thus, the one-dimensional convection-reaction system reads for $i=1, \ldots, N_{g s}$ :

$\frac{\partial}{\partial t} c_{i}(t, x)=-v \frac{\partial}{\partial x} c_{i}(t, x)+r_{i}(t, x, \mathbf{c}),(t, x) \in\left(0 ; t_{\max }\right) \times \Omega(t)$.
The single pore model developed in Section 2.1 is used to describe the processes within the domains $\Omega_{j}(t), j=1, \ldots, N_{p}$. This is consistent with the assumption that deposition outside of the pores can be neglected which would lead to a movement of the pore openings.

Consequently, the model equations for the CVI reactor model are split into the convection-reaction system (17) and nonlinear convection-diffusion-reaction systems within each pore, $\Omega_{j}(t), j=$ $1, \ldots, N_{p}$. Eq. (13) can directly be used being now valid for $(t, x) \in$ $\left(0 ; t_{\max }\right) \times \Omega_{j}(t)$ with $j=1, \ldots, N_{p}$.

The coupling between the convection-reaction system and the nonlinear convection-diffusion-reaction systems for the different pores is realised by Dirichlet boundary conditions at the pore entrances: the values of the concentrations determined at the positions of the pore entrances yield the boundary values for the concentrations within the pores. Thus, for each pore, the right-hand side of the first equation in (14) is changed to

$c_{i}(t, x=0)=\tilde{c}_{i}(t), \quad t \geqslant 0, i=1, \ldots, N_{g s}$,

where $\tilde{c}_{i}(t)$ additionally depends on the position of each of the pores within the reactor.

Boundary values for the concentrations at the reactor entrance and initial values for the concentrations within the reactor complete the CVI reactor model.

\subsection{A reduced reaction scheme as chemical model}

Using methane as a precursor in CVI, the whole hydrocarbon chemistry with many hundred of species has to be considered. Moreover, the values of the different reaction rates may vary by many orders of magnitude. This is a real challenge for modelling and numerical simulations.

To simplify the task, a reduced reaction scheme is used by creating species classes that contain different species with similar properties. Relying on the schemes proposed by Becker and Hüttinger (1998), we consider a reaction scheme with $N_{g s}=4$ gas phase and one surface species, the solid pyrolytic carbon. We split this reaction scheme in homogeneous gas phase reactions and heterogeneous surface reactions. The homogeneous gas phase reactions describe the decomposition of methane and the subsequent formation of higher hydrocarbons, whereas the latter results in the formation of solid pyrolytic carbon. With $C_{n}$ denoting the species class of hydrocarbons containing $n$ carbon atoms for $n=1,2$, the gas phase reactions are

$\mathrm{CH}_{4} \stackrel{k_{12}}{\longrightarrow} \mathrm{C}_{1} \stackrel{k_{23}}{\longrightarrow} \mathrm{C}_{2} \stackrel{k_{34}}{\longrightarrow} \mathrm{C}_{\geqslant 6}$.

Within the species class $\mathrm{C}_{\geqslant 6}$ benzene and polyaromatic hydrocarbons are assembled. The rate constants $k_{i j}$ have a unit of [ $\left.\mathrm{s}^{-1}\right]$. As can be read off from Eq. (19), $k_{12}$, for example, denotes the reaction rate for the formation of the $C_{1}$ species class from methane.

Denoting solid pyrolytic carbon by $\mathrm{C}_{\infty}$, the heterogeneous surface reactions read

$\mathrm{C}_{1} \stackrel{k_{25}}{\longrightarrow} \mathrm{C}_{\infty}$

$\mathrm{C}_{2} \stackrel{k_{35}}{\longrightarrow} \mathrm{C}_{\infty}$,

$\mathrm{C}_{\geqslant 6} \stackrel{k_{45}}{\rightarrow} \mathrm{C}_{\infty}$.

All reactions between the species classes are assumed to be of first order yielding reaction rates being linear in the concentrations of the species and independent of the pressure. The rate constants $k_{i 5}$ with $i=2,3,4$ of the heterogeneous reactions (unit: $\left[\mathrm{m} \mathrm{s}^{-1}\right]$ ) in Eq. (20) depend on the local ratio of volume to surface area:

$k_{i 5}(T)=k_{i 5}^{*}(T) \frac{V(t, x)}{A(t, x)}$ 
Table 1

Values of the rate constants of reaction scheme Eq. (19) fitted to experimental data for $T=1373.15 \mathrm{~K}$ and $p=10 \mathrm{kPa}$

\begin{tabular}{lc}
\hline Reaction $i j$ & $k_{i j}\left(\mathrm{~s}^{-1}\right)$ \\
\hline 12 & 0.71527076 \\
23 & 276.03689 \\
34 & 3.650991 \\
\hline
\end{tabular}

Table 2

Values of the rate constants of reaction scheme Eq. (20) fitted to experimental data for $T=1373.15 \mathrm{~K}$ and $p=10 \mathrm{kPa}$

\begin{tabular}{ll}
\hline Reaction $i j$ & $k_{i j}^{*}\left(\mathrm{~s}^{-1}\right)$ \\
\hline 25 & 0.40765768 \\
35 & 0.59139489 \\
45 & 0.29912904 \\
\hline
\end{tabular}

with $V(t, x)$ denoting the local volume and $A(t, x)$ the local surface area. In case of the cylindrical geometry of the pores, this ratio is given by $d_{p}(t, x) / 4$. $k_{25}$ e.g. is the rate constant for the formation of solid pyrolytic carbon from the gas phase species class $C_{1} \cdot k_{i 5}^{*}$ for $i=2,3,4$ can then be treated in the same way as the rate constants of the homogeneous gas phase reactions, $k_{i j}$. According to the chemical reactions in (19) and (20), the vector of the reaction rates of the gas phase species in Eqs. (13) and (17) is given by

$$
\left(\begin{array}{c}
r_{\mathrm{CH}_{4}}(t, x) \\
r_{\mathrm{C}_{1}}(t, x) \\
r_{\mathrm{C}_{2}}(t, x) \\
r_{\mathrm{C}_{\geqslant 6}}(t, x)
\end{array}\right)=\left(\begin{array}{c}
-k_{12} c_{\mathrm{CH}_{4}}(t, x) \\
k_{12} c_{\mathrm{CH}_{4}}(t, x)-\left(k_{23}+k_{25}^{*}\right) c_{\mathrm{C}_{1}}(t, x) \\
k_{23} c_{\mathrm{C}_{1}}(t, x)-\left(k_{34}+k_{35}^{*}\right) c_{\mathrm{C}_{2}}(t, x) \\
k_{34} c_{\mathrm{C}_{2}}(t, x)-k_{45}^{*} c_{\mathrm{C}_{\geqslant 6}}(t, x)
\end{array}\right)
$$

Using Eqs. (20), (21) and (12), the total deposition rate of solid pyrolytic carbon is given by

$$
\begin{aligned}
r_{\mathrm{C}_{\infty}}(t, x)= & k_{25}(T) c_{\mathrm{C}_{1}}(t, x)+k_{35}(T) c_{\mathrm{C}_{2}}(t, x)+k_{45}(T) c_{\mathrm{C}_{\geqslant 6}}(t, x) \\
= & \frac{d_{p}^{0}-2 h(t, x)}{4}\left(k_{25}^{*}(T) c_{\mathrm{C}_{1}}(t, x)+k_{35}^{*}(T) c_{\mathrm{C}_{2}}(t, x)\right. \\
& \left.+k_{45}^{*}(T) c_{\mathrm{C}_{\geqslant 6}}(t, x)\right)
\end{aligned}
$$

which is to be inserted into Eq. (11). The temperature dependence of the rate constants of the gas phase reactions in Eq. (20) and of $k_{i 5}^{*}(T)$ is governed by an Arrhenius law:

$k_{i j}(T)=A_{i j} \exp \left\{-\frac{E_{i j}^{a}}{R T}\right\}, \quad k_{i 5}^{*}(T)=A_{i 5}^{*} \exp \left\{-\frac{E_{i 5}^{a *}}{R T}\right\}$.

The values of the preexponential factors $A_{i j}$ and $A_{i j}^{*}$ and the activation energies $E_{i j}^{a}$ and $E_{i j}^{a *}$ were obtained by parameter fits using data of gas phase analyses of CVD experiments at pressures around $10 \mathrm{kPa}$. The resulting values of the rate constants both for the gas phase reactions in Eq. (19) and the surface reactions in Eq. (20) can be found in Tables 1 and 2 , respectively.

The values of the binary diffusion coefficients of methane and of the species classes $C_{1}$ and $C_{2}$ were calculated according to Eq. (4), whereas the value of the binary diffusion coefficient of species class $C \geqslant 6$ was estimated (Zhang and Hüttinger, 2001) (see Table 3).

The improvement using the chemical models (19) and (20) is based on the fact that no longer one direct reaction from methane to the solid carbon is assumed, but more realistically, the formation of solid carbon involves intermediate gas phase species. Additionally, different pathways for the deposition of the solid pyrolytic carbon from different gas phase species are taken into account.

Now we want to state in detail why the deposition on the pore ground can be neglected with respect to the deposition on the pore
Table 3

Values of the binary diffusion coefficients of the different species of reaction scheme Eq. (19) for $T=1373.15 \mathrm{~K}$ and $p=10 \mathrm{kPa}$

\begin{tabular}{llll}
\hline$i$ & Species & $D_{i}^{\text {bin }}\left(\mathrm{m}^{2} \mathrm{~s}^{-1}\right)$ & \\
\hline 1 & $\mathrm{CH}_{4}$ & 0.00316 & \\
2 & $\mathrm{C}_{1}$ & 0.003212 & \\
3 & $\mathrm{C}_{2}$ & 0.0025 & (estimated), Zhang and Hüttinger (2001) \\
4 & $\mathrm{C}_{2} \geqslant 6$ & 0.0006 & ( \\
\hline
\end{tabular}

walls for the geometry chosen. In complete analogy to the deposition on the pore wall deposition on the pore ground can be described. This would, however, require a two-dimensional formulation of the model, introducing the additional coordinate $r$ in radial direction. The pore length would then depend on both $t$ and $r: l=l(t, r)$. For the temporal change of the length $l(t, r)$ we would not need Eq. (2), but an equation similar to Eq. (11):

$\frac{\partial l}{\partial t}(t, r)=\frac{M_{\mathrm{C}_{\infty}}}{\varrho_{\mathrm{C}_{\infty}}} \cdot \kappa(t, x, r) \cdot r_{\mathrm{C}_{\infty}}^{\text {ground }}(t, x, r, \mathbf{c})$,

where the curvature term $\kappa(t, x, r)$ is now to be given for $l(t, r)$ analogous as for $h(t, x)$ in Eq. (11). For the case of deposition on the ground, the total deposition rate would not look like Eq. (23), but rather (the dependencies of the rate constants and of the concentrations are suppressed here)

$$
\begin{aligned}
& r_{\mathrm{C}_{\infty}}^{\text {ground }}(t, x, r, \mathbf{c})=k_{25}{ }^{c C_{1}}+k_{35}{ }^{c_{C_{2}}}+k_{45}{ }^{c_{C}}{ } \geqslant 6 \\
& =l(t, r)\left(k_{25}^{*}{ }^{c} C_{1}+k_{35}^{*}{ }^{c} C_{2}+k_{45}^{*}{ }^{*} C_{\geqslant 6}\right) \text {. }
\end{aligned}
$$

The basic criteria for neglection of deposition is: how much does the length or height of the gas phase domain relatively change due to the deposited volume on the pore ground $V_{\text {deposited }}^{\text {ground }}$ and on the pore walls $V_{\text {deposited }}^{\text {wall }}$ resp., during one time increment? Using Eqs. (11), (23), (25), (26) and the assumption about the small aspect ratio of the pores, this results in

$$
\frac{1}{l_{0}} \frac{\partial}{\partial t} V_{\text {deposited }}^{\text {ground }} / \frac{1}{d_{p}^{0}} \frac{\partial}{\partial t} V_{\text {deposited }}^{\text {wall }} \approx \frac{d_{p}^{0}}{l_{0}} \ll 1
$$

and justifies the neglection of the deposition on the pore ground. Consequently, the one-dimensional formulation proposed above is sufficient and the temporal change of the domain of the gas phase is determined by Eq. (2). Note that this formulation is one-dimensional, but the results apply to a two-dimensional case. As the numerical algorithm shows, there is no need for a discretisation in the radial direction (the height of the carbon layer is exactly computed) reducing significantly the numerical effort.

\subsection{Numerical solution}

The nonlinear system of Eq. (13) together with Eqs. (11) and (23) of the single pore model is solved numerically using the method of finite differences by approximating the spatial derivatives with central difference quotients. To introduce the notation we define for $i=1, \ldots, N_{g s}$

$c_{i}^{(m, n)}:=c_{i}(m \Delta t, n \Delta x), \quad 0 \leqslant m \leqslant M, 0 \leqslant n \leqslant N$,

$h^{(m, n)}:=h(m \Delta t, n \Delta x), \quad 0 \leqslant m \leqslant M, 0 \leqslant n \leqslant N$,

with $M, N \in \mathbb{N}$ and $\Delta t=t_{\max } / M$ and $\Delta x=l_{0} / N$.

Solving it in its fully coupled form would result in the use of forward difference quotients for the time derivatives yielding explicit difference schemes (because of the nonlinearities). Consequently, very small time steps would have to be used in order to guarantee 
the numerical stability of the algorithm. This would finally lead to greater CPU times needed to obtain the solution since at each time step inner iterations have to be performed additionally due to the nonlinearity of the system.

In contrast, the system is decoupled in the following way. At each time step, the nonlinear system of Eq. (13) is solved for the concentrations using known values for the height of the carbon layer at the actual time step. The solution is obtained by using backward Euler discretisations for the time derivatives yielding an implicit difference scheme and a linear system for the concentrations at the different points at the next time step.

For $0<m \leqslant M, 0<n<N$ and for $i=1, \ldots, N_{g s}$ we obtain using Eq. (23):

$$
\begin{aligned}
\frac{c_{i}^{(m, n)}-c_{i}^{(m-1, n)}}{\Delta t} & \\
= & \frac{2 M_{C_{\infty}}}{\varrho C_{\infty}} \frac{k_{25}^{*} c_{C_{1}}^{(m-1, n)}+k_{35}^{*} c_{C_{2}}^{(m-1, n)}+k_{45}^{*} c_{C_{\geqslant}}^{(m-1, n)}}{\sqrt{4+(\Delta x)^{-2}\left(h^{(m-1, n+1)}-h^{(m-1, n-1))^{2}}\right.} c_{i}^{(m, n)}} \\
& +6 D_{i}^{\text {bin }} \frac{X+d_{p}^{0}-4 h^{(m-1, n)}}{\left(X-2 h^{(m-1, n)}\right)^{2}} \frac{h^{(m-1, n+1)}-h^{(m-1, n-1)}}{2 \Delta x} \\
& \times \frac{c_{i}^{(m, n+1)}-c_{i}^{(m, n-1)}}{2 \Delta x} \\
& +3 D_{i}^{\text {bin }} \frac{d_{p}^{0}-2 h^{(m-1, n)}}{X-2 h^{(m-1, n)}} \frac{c_{i}^{(m, n+1)}-2 c_{i}^{(m, n)}+c_{i}^{(m, n-1)}}{(\Delta x)^{2}} \\
& +r_{i}\left(m \Delta t, n \Delta x, \mathbf{c}^{(m, n)}\right)
\end{aligned}
$$

with $X=3 D_{i}^{\text {bin }} \sqrt{\pi M_{i} / 8 R T}+d_{p}^{0}$ and

$$
\begin{aligned}
\frac{1}{\Delta t}( & \left.h^{(m, n)}-h^{(m-1, n)}\right) \\
= & \frac{M_{C_{\infty}}}{4 \varrho_{C_{\infty}}}\left(d_{p}^{0}-2 h^{(m-1, n)}\right) \\
& \times\left(1+\left(\frac{h^{(m-1, n+1)}-h^{(m-1, n-1)}}{2 \Delta x}\right)^{2}\right)^{-1 / 2} \\
& \times\left(k_{25}^{*}(T) c_{C_{1}}^{(m, n)}+k_{35}^{*}(T) c_{C_{2}}^{(m, n)}+k_{45}^{*}(T) c_{C_{\geqslant 6}}^{(m, n)}\right) .
\end{aligned}
$$

Though, in one spatial dimension, the dimensions of these resulting linear, asymmetric, and sparse systems are not yet too big, a fast and efficient method being suitable for bigger systems in future and allowing a parallel implementation was searched for. We choose a restarted version of the GMRES (Saad and Schultz, 1986) solver with an incomplete LU factorisation as preconditioner. Afterwards, these values of the concentrations are used to calculate the new height of the carbon layer by an explicit difference scheme for the combination of Eqs. (11) and (23). Based on the new values of the height of the carbon layer, updates of the values of the effective diffusion coefficients and the domain of the gas phase are performed.

In case of the CVI reactor model, the algorithm is based on the decomposition of the whole domain of the gas phase Eq. (16). First the concentrations in the gas phase domain outside of the pores are calculated by solving the discretised version of the convection-reaction system Eq. (17) using finite differences. The concentrations yield the boundary conditions for the different systems of equations of the pores that are subsequently solved independently of one another in the way described above.

The implementation was performed within an object-oriented framework in $\mathrm{C}++$ using the freely available numerical library SparseLib + + (Dongarra et al., 1994).

\section{Results and discussion}

\subsection{Results for the single pore model}

The evolution of the moving boundary $\Gamma(t)$ is evaluated for the case of two different pore geometries-pore diameter of $d_{p}^{0}=0.001 \mathrm{~m}$ and pore lengths $l_{0}=0.03 \mathrm{~m}$, and $l_{0}=0.12 \mathrm{~m}$-and for different values of temperature and pressures. As boundary conditions, pure methane was chosen with the appropriate concentration calculated using the ideal gas law.

For different infiltration times the deposition profiles within the pores are shown in Fig. 5 . In the case of $T=1373 \mathrm{~K} ; p=10 \mathrm{kPa}$ (see Fig. 5 above) increasing heights of the layer within all pores from the entrance to the ground occur. This means that continuing the infiltration process will theoretically lead to complete infiltration of all pores.

Increasing the pressure to $p=20 \mathrm{kPa}$ (see Fig. 5 middle part), the short pore still shows an increasing deposition profile as well as a higher degree of infiltration. Due to the higher pressure, the diffusion of the gas phase species is only half the value (see Eq. (4)) than in the case of $T=1373 \mathrm{~K} ; p=10 \mathrm{kPa}$. Especially the species formed in back parts of the pore will not reach the pore entrance by diffusion. This explains why in the back parts of the pores, the height of the carbon layer is higher than in the case of $T=1373 \mathrm{~K} ; p=10 \mathrm{kPa}$. The long pore closes in the middle part remaining incompletely infiltrated even after longer infiltration times since the concentrations of the higher hydrocarbons decrease due to deposition reactions before a significant part thereof could reach the depth by diffusion.

Increasing the temperature to $T=1423 \mathrm{~K}$ and keeping $p=20 \mathrm{kPa}$, the reactions become faster yielding a higher conversion of methane. Thus the height of the carbon layer increases for the short pore still showing an increasing deposition profile. At the ground the pore just starts to close. The long pore will again not be infiltrated completely; the point of closure is now closer to the entrance than in the case of $T=1373 \mathrm{~K} ; p=20 \mathrm{kPa}$ and the degree of infiltration of the long pore is even smaller (89.2-95.3\%).

Independent of the parameter constellation of $T$ and $p$ infiltration is much more efficient in early stages of the whole process and the growth velocity of the carbon layer continuously decreases.

The simulations were additionally performed for narrow pores with an initial diameter of $d_{p}^{0}=100 \mu \mathrm{m}$ (see Fig. 1) and the results did not change qualitatively and thus can also be applied to pores within a fibre felt (see Fig. 1).

In order to investigate the influence of the convective and the diffusive contribution on the right-hand side of Eq. (13) further simulations have been performed. In very narrow regions of the pore, the diffusive transport becomes negligibly small there with respect to convective transport. This is in accordance with the spatial variation of the Knudsen and of the effective diffusion coefficients found in very narrow regimes of the pores. These observations correspond to results of a time scale analysis of the dimensionless form of Eq. (13) which is for brevity not reported here.

\subsection{Influence of curvature term in the single pore model}

In order to investigate the influence of the curvature term in Eq. (13), different initial conditions for the height of the carbon layer, i.e. different initial deposition profiles, were chosen in addition to the initially flat profile. A sinus-shaped profile (for principally indicating some roughness) and a step-like profile have been considered for a pore of geometry $l_{0}=0.03 \mathrm{~m}, d_{0}=0.01 \mathrm{~m}$, and infiltration parameters $T=1373 \mathrm{~K}, p=20 \mathrm{kPa}$ (see Fig. 6).

Comparing the deposition profiles with and without consideration of the curvature term, significant relative errors in the height of the carbon layer are observed for both non-flat initial conditions 

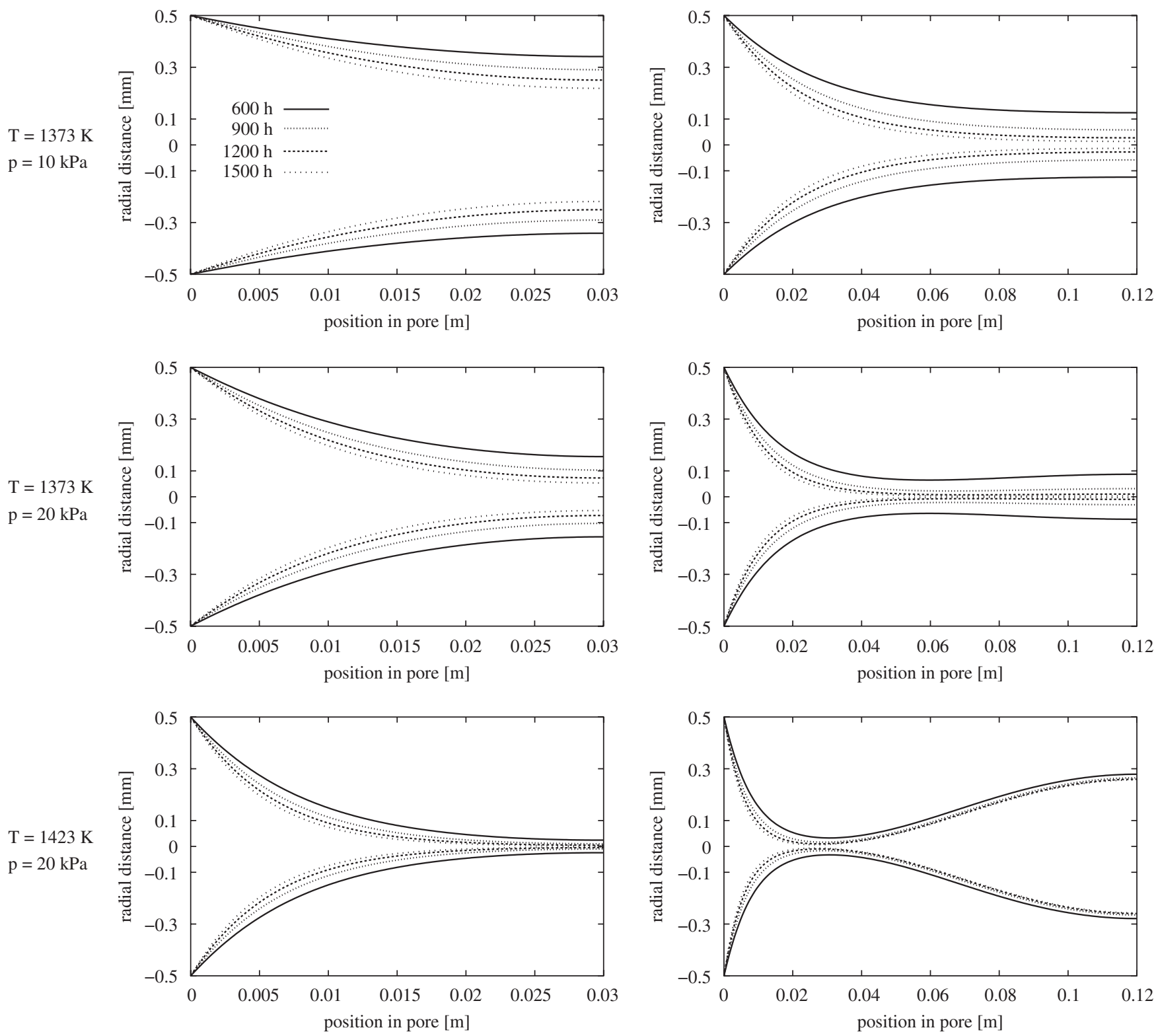

Fig. 5. Deposition profiles for different infiltration times at various process conditions for pores of $l_{0}=0.03 \mathrm{~m}$ (left) and $l_{0}=0.12 \mathrm{~m}$ (right).

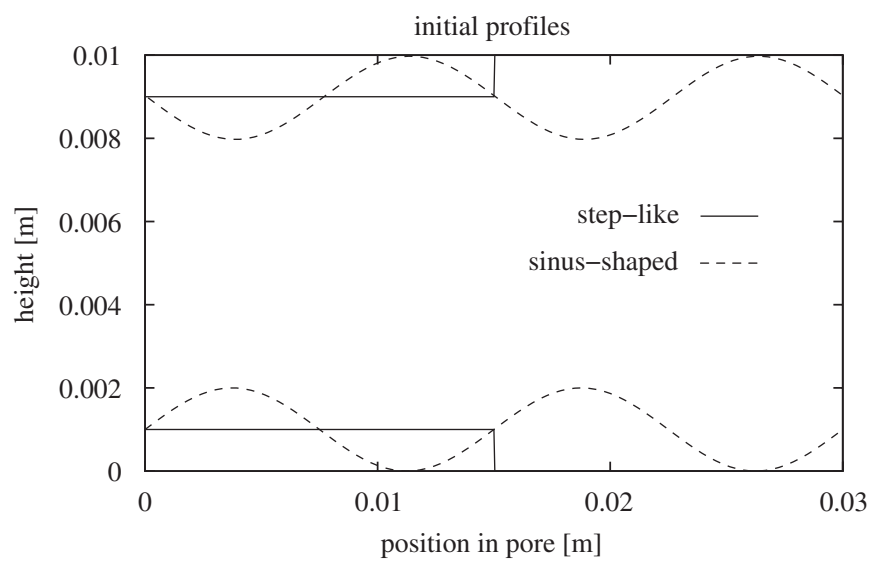

Fig. 6. Step-like and sinus-shaped initial profile within a pore. (see Figs. 7 and 8). For other pore geometries or infiltration parameters, the influence might be different. However, our numerical experiments show that neglecting the influence of curvature a priori is not correct.

Performing a complete dimensionless formulation as a basis for a rigorous time scale analysis, one ends up with the following structure of Eq. (13):

$$
\begin{aligned}
\frac{\partial}{\partial \tilde{t}} \tilde{c}_{i}(\tilde{t}, \tilde{x})= & C_{\operatorname{movb}}(\tilde{h}(\tilde{t}, \tilde{x}))\left(1+\left(\frac{d_{p}^{0}}{2 l_{0}}\right)^{2}\left(\frac{\partial \tilde{h}}{\partial \tilde{x}}\right)^{2}\right)^{-1 / 2} \\
& \times \tilde{r}_{\mathrm{C}_{\infty}}(\tilde{t}, \tilde{x}, \tilde{\mathbf{c}}) \tilde{c}_{i}(\tilde{t}, \tilde{x})+C_{\operatorname{conv}}(i, \tilde{h}(\tilde{t}, \tilde{x})) \frac{\partial \tilde{h}}{\partial \tilde{x}} \frac{\partial \tilde{c}_{i}}{\partial \tilde{x}} \\
& +C_{\operatorname{diff}}(i, \tilde{h}(\tilde{t}, \tilde{x})) \frac{\partial^{2} \tilde{c}_{i}}{\partial \tilde{x}^{2}}+\tilde{r}_{i}(\tilde{t}, \tilde{x}, \tilde{\mathbf{c}})
\end{aligned}
$$

with appropriate coefficients $C_{\text {movb }}, C_{\text {conv }}, C_{\text {diff }}$ depending all on the dimensionless height of the carbon layer and possibly on the species 


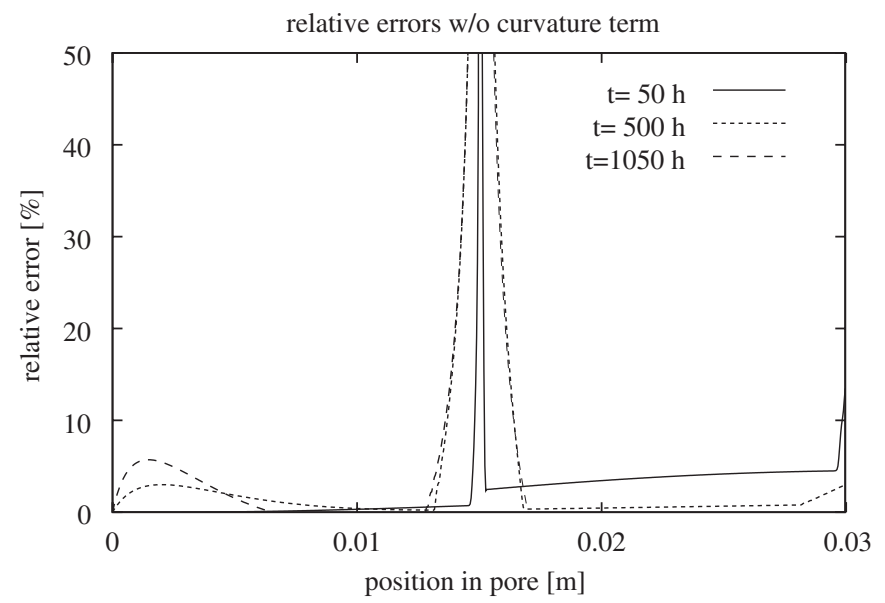

Fig. 7. Relative errors of the height of the carbon layer for different times for step-like initial profile.

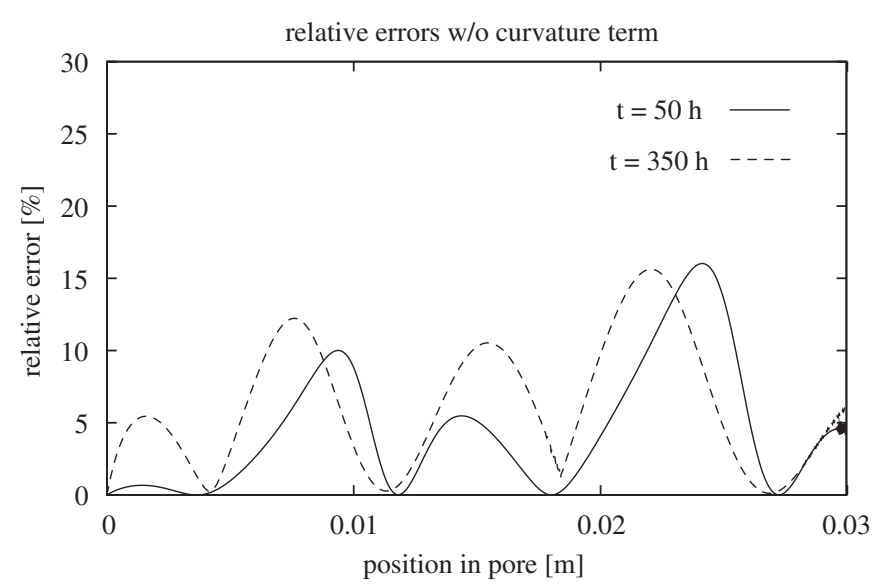

Fig. 8. Relative errors of the height of the carbon layer for different times for sinus-like initial profile.

index $i$. Note that in Eq. (31) all occurring quantities with a tilde are dimensionless. In order to estimate the relative importance of the four contributions on the right-hand side (moving boundary effects, convection-like term, diffusion, and reaction correspondingly) one can study the ratios of some of the coefficients in the early and late stages of the infiltration:

- In the early stage of infiltration with $h(t, x) \approx 0$ one has

$$
C_{\mathrm{diff}}, C_{\mathrm{conv}} \in\left(2 \times 10^{8} ; 4 \times 10^{8}\right) \text {. }
$$

- In the late stage of infiltration with $h(t, x) \approx 1$ one has

$$
C_{\mathrm{diff}} \approx 0, \quad C_{\mathrm{conv}} \in\left(2 \times 10^{8} ; 4 \times 10^{8}\right), \quad C_{\mathrm{movb}} \rightarrow \infty .
$$

The first moving boundary term on the right-hand side is qualitatively significant since it establishes the nonlinearity of the system of partial differential equations in Eqs. (11) and (13) in the manuscript with respect to the concentrations, as well. Neglecting this first term might be acceptable for some special configurations of process parameters and geometry after a rigorous check. Generally, this term has to be taken into account and cannot be simply neglected with respect to the second or the third term.

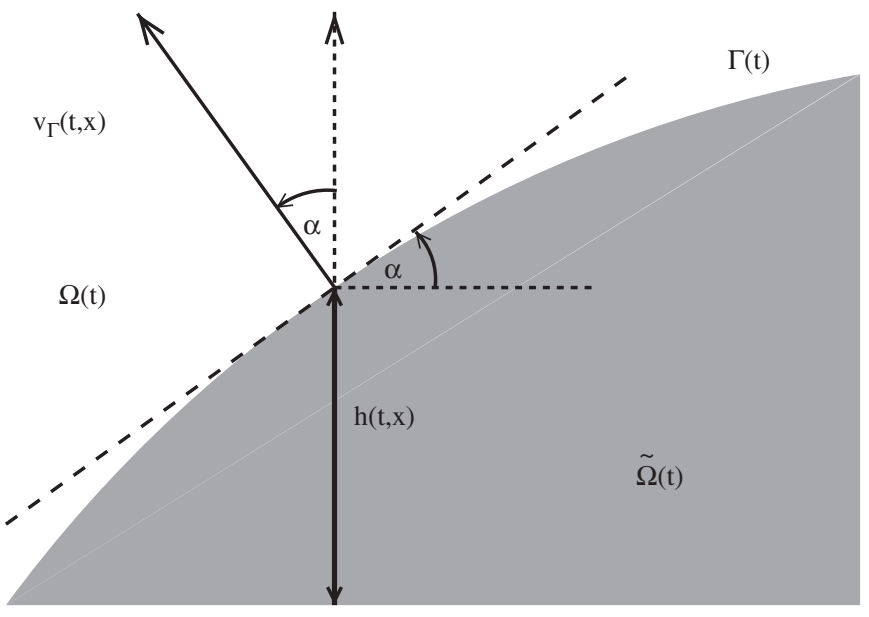

Fig. 9. Projection of the growth velocity (as a scaled clipping of Fig. 2).

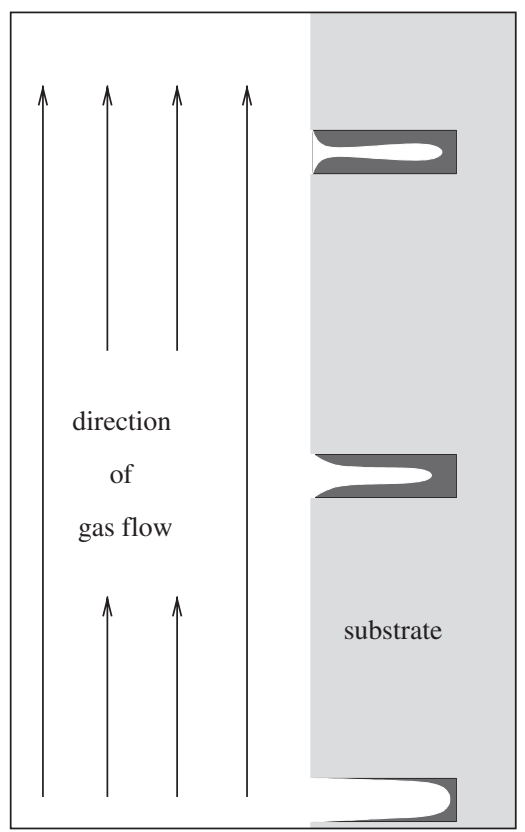

Fig. 10. Schematic view of the reactor and possible locations of the pores in the CVI reactor model.

\subsection{Results for the CVI reactor model}

The substrate is assumed to contain $N_{p}=5$ cylindrical pores, each of diameter $d_{p}^{0}=0.001 \mathrm{~m}$ that are located at distances of $0.01,0.03$, $0.05,0.07$, and $0.09 \mathrm{~m}$ from the beginning of the substrate which is taken also to be the entrance of the reactor. In Figs. 11 and 12 the deposition profiles within all pores of the substrate are shown for different pore lengths and convection velocities of the gas outside of the pores at two different infiltration times (700 and $1500 \mathrm{~h}$ ). The diameters of the pores are scaled in order to better represent the profiles. The influence of the position of the pore on the shape of the deposition profile can clearly be stated. It is a consequence of the gas phase reactions in the domain outside of the pores yielding different and time dependent compositions of the gas phase in $\Omega_{\text {out }}$ at the positions where the pores are located within the substrate (see Eq. (18)). This means that not only pure methane is present at the pore entrances, but also other hydrocarbon species that can directly form solid pyrolytic carbon by surface reactions. In Fig. 11, 

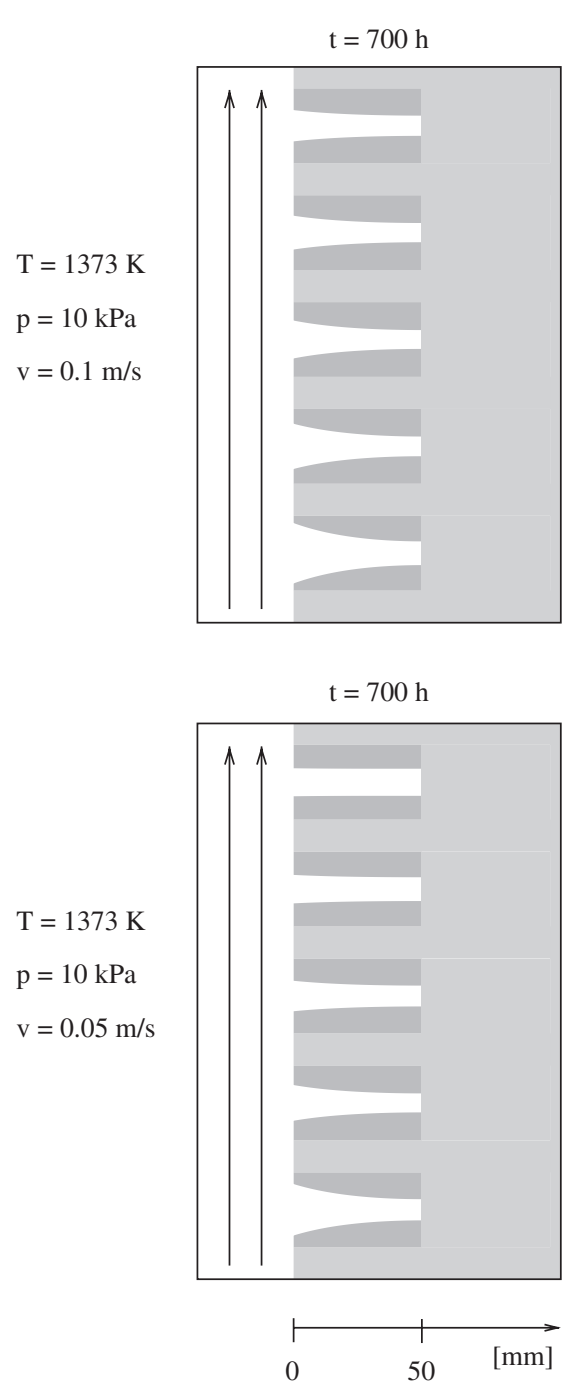
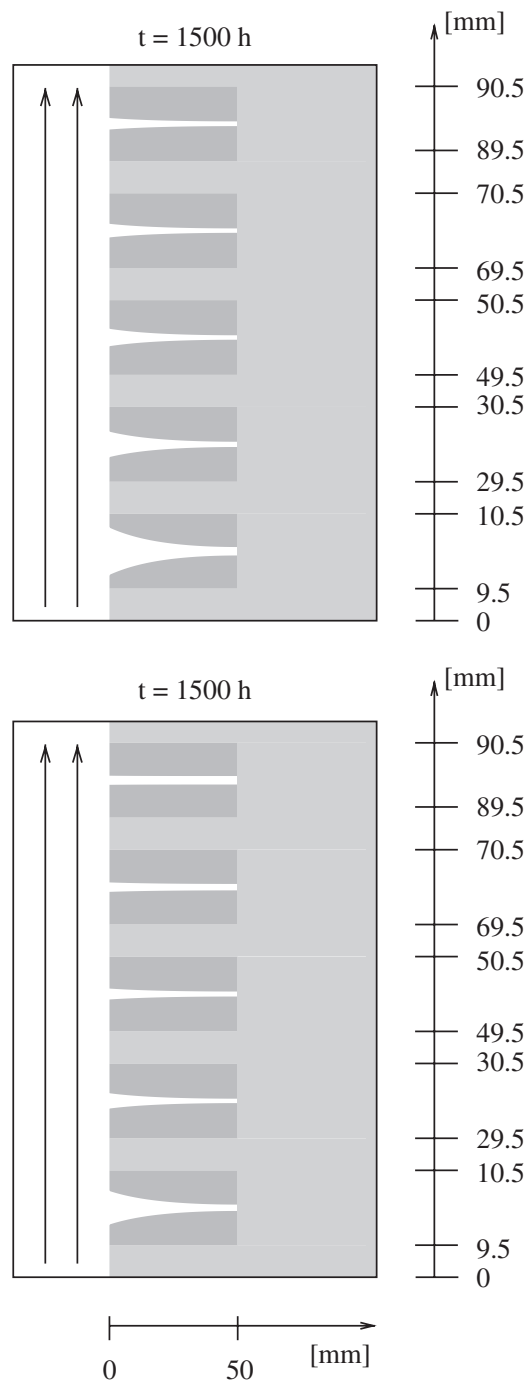

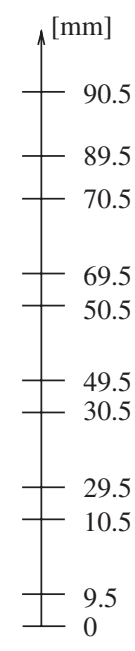

0

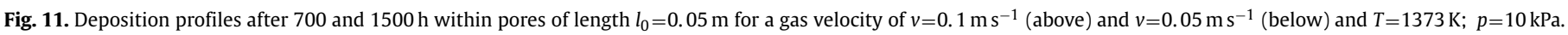

the deposition profiles within the pores mainly differ in the height of the carbon layer in the front parts of the pores, consequently. Nevertheless, the deposition profiles still increase in all pores yielding a complete infiltration if the CVI process is continued. Also in Fig. 12 the variation of the deposition profiles within the pores with the distance of the pores to the begin of the substrate are clearly shown, for both different values of the convection velocity.

Additionally, the convection velocity of the gas in the domain outside of the pores influences the deposition profiles within the pores. For different convection velocities, the composition of the gas phase at any (but fixed) position in $\Omega_{\text {out }}$ is also different. For example, this can be seen by comparing the deposition profiles within the upper pores at $t=700 \mathrm{~h}$ in Fig. 11 . More clearly, the effect can be seen in Fig. 12 especially where the deposition profiles in the upper three pores change qualitatively: in the case of $v=0.05 \mathrm{~m} \mathrm{~s}^{-1}$, the height of the carbon layer is not increasing along the position in the pore, but reaches a maximum in the front or middle parts of these pores preventing a complete infiltration of these pores for longer infiltration times.

\subsection{Comparison with available experimental data}

Hu and Hüttinger (2001) reported experimental data of CVI of pyrolytic carbon using methane as a precursor and a geometry similar to the one of the above described CVI reactor model (see Figs. 11 and 12). We adopt their reactor and substrate geometry shown in Fig. 2 in Hu and Hüttinger (2001): 9 cylindrical pores are located within a plane substrate of total length $51.5 \mathrm{~mm}$ (see Table 4). Measurements of the deposition profiles within the long pores 1 , 3,7 and 9 and the short pores 2 and 8 are available for different values of the pressure and $1373 \mathrm{~K}$. The convection velocity was $v=0.64375 \mathrm{~m} \mathrm{~s}^{-1}$ corresponding to a "residence time" of $0.08 \mathrm{~s}$. The deposition profiles were measured after an infiltration time of $150 \mathrm{~h}$ cited in $\mathrm{Hu}$ and Hüttinger (2001). In Eqs. (19) and (20) only chemical reactions of first order were used yielding reaction rates independent of the pressure. But the very complex system of chemical reactions taking place in CVI of pyrolytic carbon from methane, contains in fact higher order reactions that are pressure dependent. Since the values for the rate constants were only fitted for pressures of $p=10 \mathrm{kPa}$ (see Tables 1 and 2), the verification could only be done for $T=1373 \mathrm{~K}$ and $p=10 \mathrm{kPa}$ From Fig. 13 the good agreement between the simulated values and the measured ones is obvious. Thus the influence of the convection of the gas outside of the pores seems to be described quite well by the convection-reaction system Eq. (17). In all cases, the biggest differences occur at the points close to the pore depth. This was expected since the boundary conditions Eq. (14) at the pore depth cannot account for the effects of the end of the pore with all its geometrical discontinuities. The measured 

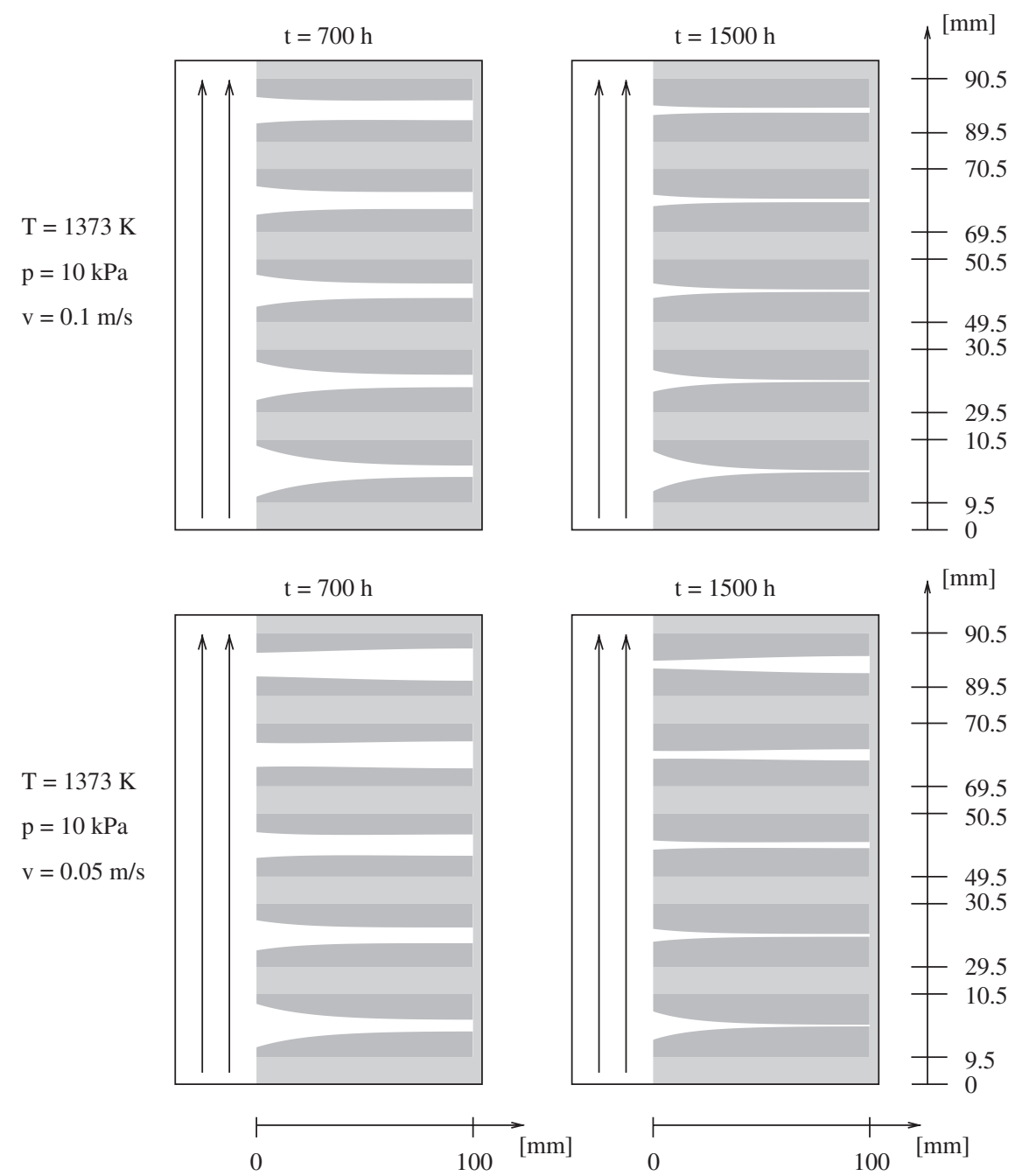

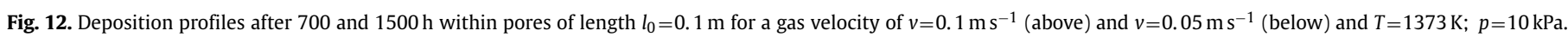

Table 4

Pore data according to $\mathrm{Hu}$ and Hüttinger (2001)

\begin{tabular}{llll}
\hline Pore number Length $(\mathrm{mm})$ & Diameter $(\mathrm{mm})$ & Distance from reactor entrance $(\mathrm{mm})$ \\
\hline 1 & 32.5 & 1.1 & 2.0 \\
2 & 17.0 & 1.1 & 7.9 \\
3 & 32.5 & 1.1 & 13.8 \\
7 & 32.5 & 1.1 & 37.4 \\
8 & 17.0 & 1.1 & 43.3 \\
9 & 32.5 & 1.1 & 49.2 \\
\hline
\end{tabular}

deposition profiles in the short pores 2 and 8 show a completely different qualitative behaviour than in all other pores, especially in regions near the pore entrances. It might be correlated to the specific flow behaviour within the first parts of these short pores at such a low pressure (Sadri and Floryan, 2002). In order to successfully compare results in these short pores with experimental data, additional contributions have to be included in the differential equations for the gas phase processes taking into account this characteristic flow behaviour.

\section{Conclusions}

A moving boundary problem for modelling chemical vapour infiltration of pyrolytic carbon is presented in this work in order to point out the interplay between transport processes, chemical reactions, and the changes in geometry due to deposition. Within a cylindrical pore, the position of the interface along the radial direction is explicitly tracked taking into account the local curvature, binary and Knudsen diffusion as well as a reduced reaction scheme instead of a single global reaction. As numerical experiments have shown, for initially non-flat profiles the influence of curvature must not be neglected. Comparison of available experimental data with the simulations of the CVI reactor model showed good agreement for low pressures.

For an optimal infiltration process of the pores within the reactor the pressure and temperature have to be carefully chosen. For higher pressures complete infiltration of long pores cannot be guaranteed. Changes in temperature affect both transport and reactive processes; thus numerical simulations are inevitable to find optimal process conditions if a given substrate geometry is to be infiltrated.

For more complex geometries, the model has to be extended to higher dimensions with the consequence of drastic increase of the numerical effort due to tracking the time dependent position of the substrate surface.

Modelling the chemical reactions, however, more precisely introduces only technical difficulties in the model proposed and adaption of the numerical strategy. This is also to be done in order to achieve accurate results for practical applications. 

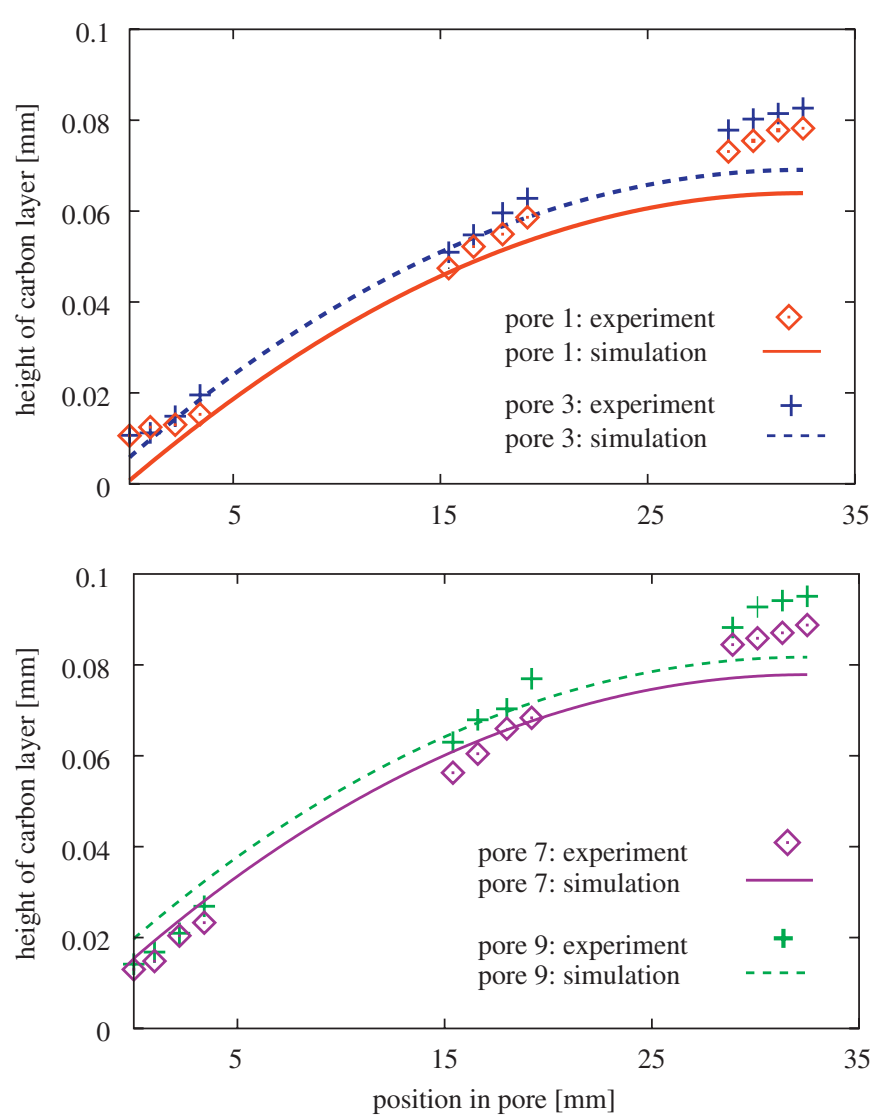

Fig. 13. Comparison between experimentally measured and simulated deposition profiles for $T=1373 \mathrm{~K}$ and $p=10 \mathrm{kPa}$ within the pores 1 and 3 (above) and pores 7 and 9 (below).

\section{Acknowledgements}

This research was performed in the Center of Excellence (Sonderforschungsbereich) 551 "Carbon from the Gas-Phase-Elementary Reactions, Structures, Materials" at Karlsruhe University. Financial support by the Deutsche Forschungsgemeinschaft is gratefully acknowledged. The authors are gratefully indebted to Dr. $\mathrm{H}$. Andrä for many stimulating discussions.

\section{References}

Andrä, H., Langhoff, T.-A., Schnack, E., Hüttinger, K.J., 2001. The role of back-mixing in the decomposition of methane. Fuel 80 (9), 1273-1277.

Becker, A., Hüttinger, K.J., 1998. Chemistry and kinetics of chemical vapour deposition of pyrocarbon-IV. Pyrocarbon deposition from methane in the low temperature regime. Carbon 36 (3), 213-224.

Benzinger, W., Becker, A., Hüttinger, K.J., 1996. Chemistry and kinetics of chemical vapour deposition of pyrocarbon-I. Fundamentals of kinetics and chemical reaction engineering. Carbon 34 (8), 957-966.

Chang, H.-C., Gottlieb, D., Marion, M., Sheldon, B.W., 1998. Mathematical analysis and optimization of infiltration processes. Journal of Scientific Computing 13 (3), 303-321.

Chung, G.Y., McCoy, B.J., Smith, J.M., Cagliostro, D.E., Carswell, M., 1991. Chemical vapor infiltration: modelling solid matrix deposition in ceramic-ceramic composites. Chemical Engineering Science 46 (3), 723-733.

Ditkowski, A., Gottlieb, D., Sheldon, B.W., 2000. On the mathematical analysis and optimization of chemical vapor infiltration in materials science. Mathematical Modelling and Numerical Analysis 34 (2), 337-351.
Domanowski, P., Kozak, J., 2001. Inverse problems of shaping by electrochemical generating machining. Journal of Materials Processing Technology 109, 347-353.

Dongarra, J., Lumsdaine, A., Pozo, R., Remington, K., 1994. A Sparse Matrix Library in C++ for High Performance Architectures. Proceedings of the Second Object Oriented Numerics Conference, pp. 214-218.

Georgiadou, M., Veyret, D., 2002. Modelling of transient electrochemical systems involving moving boundaries. Journal of the Electrochemical Society 149 (6), C324-C330.

Hou, X., Li, H., Chen, Y., Li, K., 1999. Modeling of chemical vapor infiltration process for fabrication of carbon-carbon composites by finite difference methods. Carbon 37, 669-677.

Hu, Z., Hüttinger, K.J., 2001. Chemical vapor infiltration of carbon-revised part II: experimental results. Carbon 39, 1023-1032.

Hüttinger, K.J., 1998. Chemical vapor deposition in hot wall reactors-the interaction between homogeneous gas-phase and heterogeneous surface reactions. Advanced Materials-CVD 10 (4), 151-158.

Jin, S., Wang, X., 2000. Robust numerical simulation of porosity evolution in chemical vapor infiltration I: two space dimensions. Journal of Computational Physics 162, 467-482.

Jin, S., Wang, X., 2002. Robust numerical simulation of porosity evolution in chemical vapor infiltration II: two-dimensional anisotropic fronts. Journal of Computational Physics 179, 557-577.

Jin, S., Wang, X., 2003. Robust numerical simulation of porosity evolution in chemical vapor infiltration III: three space dimensions. Journal of Computational Physics $186,582-595$

Jin, S., Wang, X., Starr, T.L., 1999. A model for front evolution with a nonlocal growth rate. Journal of Materials Research 14 (10), 3829-3832.

Jones Jr., A.D., 2006. Profile of an unsuccessful process and the criteria for a successful process for the chemical vapor infiltration process. Applied Mathematical Modelling 30, 293-306.

Katardjiev, I.V., Carter, G., Nobes, M.J., Berg, S., Blom, H.-O., 1994. Three-dimensional simulation of surface evolution during growth and erosion. Journal of Vacuum Science and Technology A 12, 61-68.

Kulik, V.I., Kulik, A.V., Ramm, M.S., Makarov, Y.N., 2004. Modeling of SiC-matrix composite formation by isothermal chemical vapor infiltration. Journal of Crystal Growth 226, 333-339.

McAllister, P., Wolf, E.E., 1991. Modelling of chemical vapor infiltration of carbon in porous carbon substrates. Carbon 29 (3), 387-396.

Merz, W., Rybka, P., 2004. A free boundary problem describing reaction-diffusion problems in chemical vapor infiltration of pyrolytic carbon. Journal of Mathematical Analysis and Applications 292, 571-588.

Middleman, S., 1989. The interaction of chemical kinetics and diffusion in the dynamics of chemical vapor infiltration. Journal of Materials Research 4 (6), 1515-1524.

Midha, V., Economou, D.J., 1998. A two-dimensional model of chemical vapor infiltration with radio-frequency heating. Journal of the Electrochemical Society 145 (19), 3569-3580.

Morell, J.I., Economou, D.J., Amundson, N.R., 1993. Chemical vapor infiltration of SiC with microwave heating. Journal of Materials Research 8 (5), 1057-1067.

Probst, K.J., Besmann, T.M., McLaughlin, J.C., Anderson, T.J., Starr, T.L., 1999. Development of a scaled-up chemical vapor infiltration system for tubular geometries. Materials at High Temperatures 16 (4), 201-205.

Reuge, N., Vignoles, G.L., 2005. Modeling of isobaric-isothermal chemical vapor infiltration: effects of reactor control parameters on a densification. Journal of Materials Processing Technology 166, 15-29.

Roman, Y.G., Kotte, J.F.A.K., deCroon, H.J.M., 1995. Analysis for the isothermal forced flow chemical vapour infiltration process. Part I: theoretical aspects. Journal of the European Ceramic Society 15 (9), 875-886.

Ross, D.S., 1988. Ion etching: an application of the mathematical theory of hyperbolic conservation laws. Journal of the Electrochemical Society 135 (5), 1235-1239.

Saad, Y., Schultz, M.H., 1986. GMRES: a generalized minimal residual algorithm for solving nonsymmetric linear systems. SIAM Journal of Scientific and Statistical Computing 7, 856-869.

Sadri, R.M., Floryan, J.M., 2002. Accurate evaluation of the loss coefficient and the entrance length of the inlet region of a channel. Journal of Fluids Engineering $124,685-693$.

Sethian, J.A., 1985. Curvature and the evolution of fronts. Communications in Mathematical Physics 101, 487-499.

Sotirchos, S.V., 1991. Dynamic modeling of chemical vapor infiltration. A.I.Ch.E. Journal 37 (9), 1365-1378.

Tago, T., Kawase, M., Hashimoto, K., 2001. Numerical simulation of the thermalgradient chemical vapor infiltration process for production of fiber-reinforced ceramic composite. Chemical Engineering Science 56 (6), 2161-2170.

Vignoles, G.L., 2006. In: Vicenzini, P. (Ed.), Advanced Fibrous Inorganic Composites V. Advances in Science and Technology 50, 97-106.

Zhang, W., Hüttinger, K.J., 2001. Chemical vapor infiltration of carbon-revised. Part I: model simulations. Carbon 39 (7), 1013-1022. 